Article

\title{
On Generalized D-Conformal Deformations of Certain Almost Contact Metric Manifolds
}

\author{
Nülifer Özdemir ${ }^{1,+} \mathbb{D}$, Şirin Aktay ${ }^{1,+}$ iD and Mehmet Solgun $2, *,+(\mathbb{D}$ \\ 1 Department of Mathematics, Eskişehir Technical University, Eskişehir 26555, Turkey; \\ nozdemir@eskisehir.edu.tr (N.Ö.); sirins@eskisehir.edu.tr (Ş.A.) \\ 2 Department of Mathematics, Bilecik Şeyh Edebali University, Bilecik 11230, Turkey \\ * Correspondence: mehmet.solgun@bilecik.edu.tr; Tel.: +90-506-244-0411 \\ + These authors contributed equally to this work.
}

Received: 20 December 2018; Accepted: 5 February 2019; Published: 13 February 2019

check for updates

\begin{abstract}
In this work, we consider almost contact metric manifolds. We investigate the generalized D-conformal deformations of nearly K-cosymplectic, quasi-Sasakian and $\beta$-Kenmotsu manifolds. The new Levi-Civita covariant derivative of the new metric corresponding to deformed nearly K-cosymplectic, quasi-Sasakian and $\beta$-Kenmotsu manifolds are obtained. Under some restrictions, deformed nearly K-cosymplectic, quasi-Sasakian and $\beta$-Kenmotsu manifolds are obtained. Then, the scalar curvature of these three classes of deformed manifolds are analyzed.
\end{abstract}

Keywords: Riemannian manifolds; almost contact metric structure; D-conformal deformation

MSC: 53C10; 53C25; 53C27

\section{Introduction}

There exist several type of deformations of almost contact metric structures. For example conformal deformations, D-homothetic deformations etc. These deformations were studied by several authors [1-4]. In [4], generalized D-conformal deformations are applied to trans-Sasakian manifolds where the covariant derivatives of the deformed metric is evaluated under the condition that the functions used in deformation depend only on the direction of the characteristic vector field of the trans-Sasakian structure. In this study, in order to simplify tedious calculations, we obtain the new covariant derivatives of deformed almost contact metric structures seperately for the cases where the characteristic vector field is parallel, Killing and the one form dual to the characteristic vector field is closed. Then, we study generalized D-conformal deformations of nearly K-cosymplectic, quasi-Sasakian and $\beta$-Kenmotsu manifolds. We analyse how the class of almost contact metric structures change. Under some restrictions, deformed nearly K-cosymplectic, quasi-Sasakian and $\beta$-Kenmotsu manifolds are obtained. In addition, scalar curvature of deformed manifolds are examined for these three classes.

\section{Preliminaries}

Let $M$ be an odd-dimensional $C^{\infty}$ manifold. An almost contact metric structure on $M$ is defined by a $(1,1)$-type tensor field $\varphi$, a vector field $\xi$, a 1 -form $\eta$ and a metric $g$ on $M$ such that

$$
\begin{gathered}
\varphi^{2}(X)=-X+\eta(X) \xi, \quad \eta(X)=1, \\
g(\varphi(X), \varphi(Y))=g(X, Y)-\eta(X) \eta(Y), \quad g(X, \xi)=\eta(X),
\end{gathered}
$$


for any vector fields $X, Y$ on $M$. A manifold equipped with an almost contact metric structure is called an almost contact metric manifold. The fundamental 2-form $\Phi$ of the almost contact metric manifold $M$ is defined by

$$
\Phi(X, Y)=g(X, \varphi(Y))
$$

for all vector fields $X, Y$ on $M$, and the form $\Phi$ satisfies $\eta \wedge \Phi^{n} \neq 0$, where dimension of $M$ is $2 n+1$. Hence, an almost contact metric manifold is orientable. In addition, the structure group of an almost contact metric manifold reduces to the group $U(n) \times 1$.

According to properties of the Levi-Civita covariant derivative of the fundamental 2-form $\Phi$, there is a classification of almost contact metric manifolds in [5]. A space having the same symmetries as the covariant derivative of the fundamental 2-form was written. This space is

$$
\begin{aligned}
\mathcal{C}=\left\{\alpha \in \otimes_{3}^{0} T_{p} M: \alpha(X, Y, Z)=\right. & -\alpha(X, Z, Y)=-\alpha(X, \varphi(Y), \varphi(Z)) \\
& +\eta(Y) \alpha(X, \xi, Z)+\eta(Z) \alpha(X, Y, \xi)\}
\end{aligned}
$$

for all $p \in M$. The space $\mathcal{C}$ was decomposed into $12 U(n) \times 1$ irreducible components, denoted by $\mathcal{C}_{1}, \ldots, \mathcal{C}_{12}$ as shown in Table 1 . There exist $2^{12}$ invariant subspaces, each corresponding to a class of almost contact metric manifolds. For example, the trivial class corresponds to the class of cosymplectic (called co-Kähler by some authors) manifolds, $\mathcal{C}_{1}$ is the class of nearly K-cosymplectic manifolds, $\mathcal{C}_{6} \otimes \mathcal{C}_{7}$ is the class of quasi-sasakian manifolds, $\mathcal{C}_{5}$ is the class of $\beta$-Kenmotsu manifolds etc [5]. Also, a similar classification was made by [6]. In this work, we will use the definitions of some other classes in the context by using the notation in $[5,6]$. According to this classification; some special classes of almost contact metric manifolds coincide with a suitable sum of some classes of $\mathcal{C}_{i}$.

When the dimension of the manifold is 3 , then $\mathcal{C}=\mathcal{C}_{5} \oplus \mathcal{C}_{6} \oplus \mathcal{C}_{9} \oplus \mathcal{C}_{12}$ [5].

Table 1. Defining relations for each of the twelve classes [5].

\begin{tabular}{ll}
\hline Classes & Defining Relations \\
\hline $\mathcal{C}_{1}$ & $\left(\nabla_{X} \Phi\right)(X, Y)=0, \nabla \eta=0$ \\
\hline $\mathcal{C}_{2}$ & $d \Phi=\nabla \eta=0$ \\
\hline $\mathcal{C}_{3}$ & $\left(\nabla_{X} \Phi\right)(Y, Z)-\left(\nabla_{\varphi(X)} \Phi\right)(\varphi(Y), Z)=0, \delta \Phi=0$ \\
\hline $\mathcal{C}_{4}$ & $\left(\nabla_{X} \Phi\right)(Y, Z)=\frac{-1}{2(n-1)}[g(\varphi(X), \varphi(Y)) \delta \Phi(Z)-g(\varphi(X), \varphi(Z)) \delta \Phi(Y)$ \\
\hline $\mathcal{C}_{5}$ & $\left(\nabla_{X} \Phi\right)(Y, Z)=\frac{1}{2 n}[\Phi(X, Z) \eta(Y)-\Phi(X, Y) \eta(Z)] \delta \eta$ \\
\hline $\mathcal{C}_{6}$ & $\left(\nabla_{X} \Phi\right)(Y, Z)=\frac{1}{2 n}[g(X, Z) \eta(Y)-g(X, Y) \eta(Z)] \delta \Phi(\xi)$ \\
\hline $\mathcal{C}_{7}$ & $\left(\nabla_{X} \Phi\right)(Y, Z)=\eta(Z)\left(\nabla_{Y} \eta\right)(\varphi(X))+\eta(Y)\left(\nabla_{\varphi(X)} \eta\right) Z, \delta \Phi=0$ \\
\hline $\mathcal{C}_{8}$ & $\left(\nabla_{X} \Phi\right)(Y, Z)=-\eta(Z)\left(\nabla_{Y} \eta\right)(\varphi(X))+\eta(Y)\left(\nabla_{\varphi(X)} \eta\right) Z, \delta \eta=0$ \\
\hline $\mathcal{C}_{9}$ & $\left(\nabla_{X} \Phi\right)(Y, Z)=\eta(Z)\left(\nabla_{Y} \eta\right)(\varphi(X))-\eta(Y)\left(\nabla_{\varphi(X)} \eta\right) Z$ \\
\hline $\mathcal{C}_{10}$ & $\left(\nabla_{X} \Phi\right)(Y, Z)=-\eta(Z)\left(\nabla_{Y} \eta\right)(\varphi(X))-\eta(Y)\left(\nabla_{\varphi(X)} \eta\right) Z$ \\
\hline $\mathcal{C}_{11}$ & $\left(\nabla_{X} \Phi\right)(Y, Z)=-\eta(X)\left(\nabla_{\xi} \Phi\right)(\varphi(Y), \varphi(Z))$ \\
\hline $\mathcal{C}_{12}$ & $\left(\nabla_{X} \Phi\right)(Y, Z)=\eta(X) \eta(Z)\left(\nabla_{\xi} \eta\right)(\varphi(Y))-\eta(X) \eta(Y)\left(\nabla_{\xi} \eta\right)(\varphi(Z))$ \\
\hline
\end{tabular}

Let $(M, \varphi, \xi, \eta, g)$ be an almost contact metric manifold. If we take

$$
\tilde{\varphi}:=\varphi, \quad \tilde{\xi}:=\frac{1}{a} \xi, \quad \tilde{\eta}:=a \eta, \quad \tilde{g}:=b g+\left(a^{2}-b\right) \eta \otimes \eta,
$$


where $a$ and $b$ are positive functions on $M$, one can easily check that $(M, \tilde{\varphi}, \tilde{\xi}, \tilde{\eta}, \tilde{g})$ is an almost contact metric manifold too. This deformation is called a generalized D-conformal deformation [4].

After this deformation, the derivation of the new fundamental 2-form $\tilde{\Phi}$ is

$$
d \tilde{\Phi}(X, Y, Z)=d \Phi(X, Y, Z)+X[b] \Phi(Y, Z)-Y[b] \Phi(X, Z)+Z[b] \Phi(X, Y) .
$$

\section{Generalized D-Conformal Deformations of Nearly K-Cosymplectic Manifolds}

Let $(M, \varphi, \xi, \eta, g)$ be a nearly K-cosymplectic manifold (that is, belongs to class $\left.C_{1}\right)$. Defining relations of this class are

$$
\nabla_{X} \xi=0 \quad \text { and } \quad\left(\nabla_{X} \varphi\right)(Y)+\left(\nabla_{Y} \varphi\right)(X)=0
$$

or equivalently

$$
\left(\nabla_{X} \Phi\right)(X, Y)=0 \text { and } \nabla \eta=0 .
$$

To calculate the new Levi-Civita covariant derivative $\widetilde{\nabla}$ of a nearly K-cosymplectic manifold after applying a generalized D-conformal deformation, we need only to consider the property that $\xi$ is parallel. Hence, we state the following lemma.

Lemma 1. Let $(M, \varphi, \xi, \eta, g)$ be an almost contact metric manifold such that characteristic vector field $\xi$ is parallel. If a generalized D-conformal deformation is applied, then the new covariant derivative of the new metric $\tilde{g}$ is obtained as

$$
\begin{aligned}
\widetilde{\nabla}_{X} Y= & \nabla_{X} Y+\frac{1}{2 b} g(\varphi(X), \varphi(Y)) \varphi^{2}(\operatorname{grad} b)+\frac{a}{b} \eta(X) \eta(Y) \varphi^{2}(\text { grad } a) \\
& +\frac{1}{a}(X[a] \eta(Y)+Y[a] \eta(X)) \xi-\frac{1}{2 b}(X[b] \eta(Y)+Y[b] \eta(X)) \xi \\
& -\frac{1}{2 a^{2}} \xi[b] g(\varphi(X), \varphi(Y)) \xi-\frac{1}{a} \xi[a] \eta(X) \eta(Y) \xi \\
& +\frac{1}{2 b}(X[b] Y+Y[b] X) .
\end{aligned}
$$

Proof. Using Kozsul's formula and that $\xi$ is parallel, one can get

$$
\begin{aligned}
& 2 b g\left(\widetilde{\nabla}_{X} Y, Z\right)+2\left(a^{2}-b\right) \eta\left(\widetilde{\nabla}_{X} Y\right) \eta(Z) \\
& =2 b g\left(\nabla_{X} Y, Z\right)+X[b] g(Y, Z)-X[b] \eta(Y) \eta(Z) \\
& +Y[b] g(X, Z)-Y[b] \eta(X) \eta(Z)-Z[b] g(X, Y)+Z[b] \eta(X) \eta(Y) \\
& +2 a X[a] \eta(Y) \eta(Z)+2 a Y[a] \eta(X) \eta(Z) \\
& -2 a Z[a] \eta(X) \eta(Y)+2\left(a^{2}-b\right) \eta\left(\nabla_{X} Y\right) \eta(Z) .
\end{aligned}
$$

Take $Z=\xi$ in the Equation (2), to obtain

$$
\begin{aligned}
\eta\left(\widetilde{\nabla}_{X} Y\right)= & \eta\left(\nabla_{X} Y\right)-\frac{1}{2 a^{2}} \xi[b] g(\varphi(X), \varphi(Y)) \\
& +\frac{1}{a}(X[a] \eta(Y)+Y[a] \eta(X))-\frac{1}{a} \xi[a] \eta(X) \eta(Y) .
\end{aligned}
$$

Hence, we write the new Levi-Civita covariant derivative of the new metric $\tilde{g}$ as in (1).

Now we show that under some restrictions, it is possible to obtain a nearly K-cosymplectic structure from an old one by a generalized D-conformal deformation. 
Theorem 1. Let $(M, \varphi, \xi, \eta, g)$ be a nearly K-cosymplectic manifold and consider a generalized D-conformal deformation on $M$ with positive functions $a$ and $b .(M, \tilde{\varphi}, \tilde{\xi}, \tilde{\eta}, \tilde{g})$ is a nearly K-cosymplectic manifold if and only if grad $a=\xi[a] \xi$ and $b$ is a constant.

Proof. If we take $Y=\tilde{\zeta}$ in (1), since $\xi$ is parallel,

$$
\widetilde{\nabla}_{X} \tilde{\xi}=\frac{1}{b}\left(\eta(X) \varphi^{2}(\operatorname{grad} a)-\frac{1}{2 a} \xi[b] \varphi^{2}(X)\right)
$$

is obtained. Also, we have $\widetilde{\nabla}_{X} \tilde{\xi}=0$ if and only if grad $a=\xi[a] \xi$ and $\xi[b]=0$. On the other hand, since $\left(\nabla_{X} \Phi\right)(X, Y)=-g\left(\left(\nabla_{X} \varphi\right)(X), Y\right)$, the relation $0=\left(\nabla_{X} \Phi\right)(X, Y)$ is equivalent to $\left(\nabla_{X} \varphi\right)(X)=0$, or by polarization

$$
\left(\nabla_{X} \varphi\right)(Y)+\left(\nabla_{Y} \varphi\right)(X)=0
$$

Then

$$
\begin{aligned}
\left(\widetilde{\nabla}_{X} \varphi\right)(Y)+\left(\widetilde{\nabla}_{Y} \varphi\right)(X)= & \left(\nabla_{X} \varphi\right)(Y)+\left(\nabla_{Y} \varphi\right)(X) \\
& +\frac{1}{a}(\varphi(Y)[a] \eta(X)+\varphi(X)[a] \eta(Y)) \xi \\
& -\frac{1}{2 b}(\varphi(Y)[b] \eta(X)+\varphi(X)[b] \eta(Y)) \xi \\
& -\frac{1}{2 b}(X[b] \varphi(Y)+Y[b] \varphi(X)-\varphi(X)[b] Y-\varphi(Y)[b] X) \\
& +\frac{1}{b} g(\varphi(X), \varphi(Y)) \varphi(\operatorname{grad} b) \\
& +\frac{2 a}{b} \eta(X) \eta(Y) \varphi(\operatorname{grada}) .
\end{aligned}
$$
a constant.

Now it is easy to see that $\left(\widetilde{\nabla}_{X} \varphi\right)(Y)+\left(\widetilde{\nabla}_{Y} \varphi\right)(X)=0$ if and only if grad $a=\xi[a] \xi$ and $b$ is

We give the following example on nearly K-cosymplectic structures.

Example 1. Let $(M, J, h)$ be an almost Hermitian manifold, dim $M=2 n$. Consider the almost contact metric structure $(\varphi, \xi, \eta, g)$ on $M \times \mathbb{R}$ where

$$
\begin{gathered}
\varphi\left(X, f \frac{d}{d t}\right)=(J X, 0), \quad \xi=\left(0, \frac{d}{d t}\right), \quad \eta\left(X, f \frac{d}{d t}\right)=f, \\
g\left(\left(X, f \frac{d}{d t}\right),\left(Y, g \frac{d}{d t}\right)\right)=h(X, Y)+f g,
\end{gathered}
$$

where $f$ and $g$ are $C^{\infty}$ functions on $M \times \mathbb{R}, X, Y$ are any vector fields on $M$. Then, $M \times \mathbb{R}$ is nearly $K$-cosymplectic if and only if $M$ is nearly Kaehlerian [5].

Consider a generalized D-conformal deformation with a $C^{\infty}$ function $a: M \times \mathbb{R} \longrightarrow \mathbb{R}, a(x, t)=F(t)>$ 0 and a positive constant $b$. Since the function a depends only on $t$, grada $=\xi[a]$. Then, by Theorem 1 , the new almost contact metric structure on $M \times \mathbb{R}$ is also nearly K-cosymplectic.

Let $(M, \varphi, \xi, \eta, g)$ be an almost contact metic manifold and $\left\{e_{1}, e_{2}, \cdots, e_{2 n}, \xi\right\}$ be a local orthonormal frame for the metric $g$ on $M$. Then, after a generalized D-conformal deformation, $\left\{\tilde{e}_{1}, \tilde{e}_{2}, \cdots, \tilde{e}_{2 n}, \tilde{\xi}\right\}$ is a local orthonormal frame for the metric $\tilde{g}$, where $\tilde{e}_{i}=\frac{1}{\sqrt{b}} e_{i}$ and $\tilde{\xi}=\frac{1}{a} \xi$, for $i=1,2, \cdots, 2 n$. If $(M, \varphi, \xi, \eta, g)$ is a nearly $\mathrm{K}$-cosymplectic manifold, $b$ is a positive constant and 
grada $=\xi[a] \xi$, then the new almost contact metric structure is nearly K-cosymplectic and the new Levi-Civita covariant derivative is written as

$$
\tilde{\nabla}_{X} Y=\nabla_{X} Y+\frac{1}{a} \xi[a] \eta(X) \eta(Y) \xi .
$$

By direct calculation, one can get the new curvature tensor $\tilde{R}$, Ric operator $\tilde{Q}$ and scalar curvature $\tilde{S}$ as

$$
\begin{gathered}
\tilde{R}(X, Y) Z=R(X, Y) Z+\frac{1}{a} X[\xi[a]] \eta(Y) \eta(Z) \xi+\frac{1}{a} Y[\xi[a]] \eta(X) \eta(Z) \xi \\
\tilde{Q}(X)=\frac{1}{b} Q(X), \\
\tilde{S}=\frac{1}{b} S .
\end{gathered}
$$

Thus, one can obtain the following theorem.

Theorem 2. Let $(M, \varphi, \xi, \eta, g)$ be a nearly K-cosymplectic manifold whose scalar curvature $S$ is constant. If $b=S$ and grada $=\xi[a] \xi$, then new nearly $K$-cosymplectic manifold $(M, \tilde{\varphi}, \tilde{\xi}, \tilde{\eta}, \tilde{g})$ is locally isometric to the sphere. In addition, if $b=1$ and $a$ is a function such that grada $=\xi[a] \xi$, the manifolds $(M, \varphi, \xi, \eta, g)$ and $(M, \tilde{\varphi}, \tilde{\xi}, \tilde{\eta}, \tilde{g})$ are locally isometric.

In addition, since $\delta \eta=0$ and $\delta \Phi=0$, one can express the new coderivation of the new fundamental 1-form $\tilde{\eta}$ and 2-form $\widetilde{\Phi}$ as

$$
\begin{aligned}
\widetilde{\delta} \tilde{\eta}= & -\sum_{i=1}^{2 n}\left(\widetilde{\nabla}_{\tilde{e}_{i}} \tilde{\eta}\right)\left(\tilde{e}_{i}\right)=\frac{a}{b}\left(\delta \eta-\frac{n}{a^{2}} \xi[b]\right)=-\frac{n}{a b} \xi[b] \\
(\widetilde{\delta} \tilde{\Phi})(X) & =-\sum_{i=1}^{2 n}\left(\widetilde{\nabla}_{\tilde{e}_{i}} \tilde{\Phi}\right)\left(\tilde{e}_{i}, X\right)-\left(\widetilde{\nabla}_{\tilde{\xi}} \tilde{\Phi}\right)(\tilde{\xi}, X) \\
& =\delta \Phi+\frac{1}{2 b} g(\operatorname{grad} b, \varphi(X))-\frac{1}{a} g(\operatorname{grad} a, \varphi(X)) \\
& =\frac{n-1}{b} g(\varphi(\operatorname{grad} b), X)+\frac{1}{a} g(\varphi(\operatorname{grad} a), X) \\
& =g\left(\varphi\left(\operatorname{grad} \ln \left(b^{n-1} a\right)\right), X\right) .
\end{aligned}
$$

Hence, one can conclude that, if $(M, \varphi, \xi, \eta, g)$ is nearly $\mathrm{K}$-cosymplectic, then $(M, \tilde{\varphi}, \tilde{\xi}, \tilde{\eta}, \tilde{g})$ is a semi-cosymplectic manifold if and only if $\xi[b]=0$ and $\operatorname{grad}\left(\ln \left(b^{n-1} a\right)\right)=g\left(\xi, \operatorname{grad}\left(\ln \left(b^{n-1} a\right)\right) \xi\right.$.

\section{Generalized D-Conformal Deformations of Quasi-Sasakian Manifolds}

In this case, we consider generalized D-conformal deformations of quasi-Sasakian manifolds. An almost contact metric manifold is called quasi-Sasakian if it is normal and its fundamental 2-form $\Phi$ is closed, that is,

$$
[\varphi, \varphi](X, Y)+d \eta(X, Y) \xi=0 \text { and } d \Phi=0,
$$

for all vector field $X$ and $Y$. Quasi-Sasakian manifolds are the class $\mathcal{C}_{6} \oplus \mathcal{C}_{7}$. The most important feature of a quasi-Sasakian manifold is that the fundamental vector field $\xi$ is a Killing vector field [7].

Let $(M, \varphi, \xi, \eta, g)$ be an almost contact metric manifold such that $\xi$ is Killing. The Levi-Civita covariant derivative of $\widetilde{g}$ is calculated using Kozsul's formula, only by considering that $\tilde{\xi}$ is Killing. 
Lemma 2. Let $(M, \varphi, \xi, \eta, g)$ be an almost contact metric manifold such that characteristic vector field $\xi$ is Killing. If a generalized D-conformal deformation is applied, then the new covariant derivative of the new metric $\tilde{g}$ is obtained as

$$
\begin{aligned}
\widetilde{\nabla}_{X} Y= & \frac{\left(a^{2}-b\right)}{2 a^{2} b} \xi[b] g(\varphi(X), \varphi(Y)) \xi-\frac{1}{2 b}\left(X[b] \varphi^{2}(Y)+Y[b] \varphi^{2}(X)\right) \\
& -\frac{1}{2 b} g(\varphi(X), \varphi(Y)) g r a d b+\frac{1}{a}[X[a] \eta(Y)+Y[a] \eta(X)] \xi \\
& +\nabla_{X} Y+\frac{\left(a^{2}-b\right)}{a b} \xi[a] \eta(X) \eta(Y) \xi \\
& -\frac{a}{b} \eta(X) \eta(Y) \text { grada }+\frac{\left(a^{2}-b\right)}{b}\left[\eta(Y) \nabla_{X} \xi+\eta(X) \nabla_{Y} \xi\right] .
\end{aligned}
$$

Now our aim is to obtain a quasi-Sasakian manifold after applying a generalized D-conformal deformation to a quasi-Sasakian manifold. First we give the condition for $\widetilde{\xi}$ to be Killing with respect to $\tilde{g}$.

Lemma 3. $\widetilde{\xi}$ is Killing vector field if and only if $\xi[b]=0$ and grada $=\xi[a] \xi$.

Proof. Let $\widetilde{\zeta}$ be a Killing vector field. Then

$$
\mathcal{L}_{\widetilde{\xi}} \tilde{g}(X, Y)=0,
$$

for all vector fields $X, Y$. Since

$$
\begin{aligned}
\mathcal{L}_{\tilde{\xi}} \tilde{g}(X, Y)= & \frac{1}{a} \xi[b] g(\varphi(X), \varphi(Y))+2 \xi[a] \eta(X) \eta(Y) \\
& -X[a] \eta(Y)-Y[a] \eta(X),
\end{aligned}
$$

if we take $X=\xi$ in (9), we obtain

$$
\xi[a] \eta(Y)=Y[a]
$$

hence

$$
\text { grada }=\xi[a] \xi .
$$

In addition, if we take grada $=\xi[a] \xi$ in (9), we get $\xi[b]=0$. Converse of the lemma is trivial.

We can obtain quasi-Sasakian manifolds by deforming the old ones as follows.

Theorem 3. Let $(M, \varphi, \xi, \eta, g)$ be a quasi-Sasakian manifold and consider a generalized D-conformal deformation with positive functions $a$ and $b$. Then, the new almost contact metric manifold obtained by generalized D-conformal deformation is quasi-Sasakian if and only if $b$ is a constant and a depends only on the direction of $\xi$.

Proof. Consider a generalized D-conformal deformation on $M$ with positive functions $a$ and $b$. Let $(M, \tilde{\varphi}, \tilde{\xi}, \tilde{\eta}, \tilde{g})$ be a quasi-Sasakian manifold. Then, $\tilde{\xi}$ is a Killing vector field. Thus from Lemma 3, we get

$$
\xi[b]=0 \text { and grada }=\xi[a] \xi .
$$

Conversely, let $b$ be a constant and grad $a=\xi[a] \xi$. By Lemma 3, $\tilde{\xi}$ is Killig. In addition, since a quasi-Sasakian manifold is normal, we have

$$
[\varphi, \varphi](X, Y)+d \eta(X, Y) \xi=0
$$


Then

$$
\begin{aligned}
{[\widetilde{\varphi}, \widetilde{\varphi}](X, Y)+d \widetilde{\eta}(X, Y) \widetilde{\xi} } & =[\varphi, \varphi](X, Y)+\frac{1}{a} d \widetilde{\eta}(X, Y) \xi \\
& =[\varphi, \varphi](X, Y)+d \eta(X, Y) \xi+\frac{1}{a}(X[a] \eta(Y)-Y[a] \eta(X)) \xi
\end{aligned}
$$

Since grad $a=\xi[a] \xi$, the equation

$$
[\widetilde{\varphi}, \widetilde{\varphi}](X, Y)+d \widetilde{\eta}(X, Y) \widetilde{\xi}=0
$$

is satisfied. Also, for a quasi-Sasakian manifold $d \Phi=0$, thus we get

$$
d \tilde{\Phi}(X, Y, Z)=X[b] \Phi(Y, Z)-Y[b] \Phi(X, Z)+Z[b] \Phi(X, Y)
$$

Since the function $b$ is a constant, we obtain $d \tilde{\Phi}(X, Y, Z)=0$. As a result $(M, \tilde{\varphi}, \tilde{\xi}, \tilde{\eta}, \tilde{g})$ is a quasi-Sasakian manifold.

In addition, one can obtain the following corollary:

Corollary 1. Let $(M, \varphi, \xi, \eta, g)$ be a quasi-Sasakian manifold. If a is a positive function such that grad a= $\xi[a] \xi$ and $b$ is a positive constant, then the new almost contact metric manifold $(M, \tilde{\varphi}, \tilde{\xi}, \tilde{\eta}, \tilde{g})$ is normal.

If $(M, \varphi, \xi, \eta, g)$ is a quasi-Sasakian manifold and $a$ and $b$ are positive functions such that $\xi[b]=0$ ( $b$ need not be constant) and grada $=\xi[a] \xi$, then in new almost contact metric manifold $(M, \tilde{\varphi}, \tilde{\xi}, \tilde{\eta}, \tilde{g})$, one can compute directly coderivation of $\widetilde{\delta} \tilde{\eta}$ and $\widetilde{\delta} \tilde{\Phi}$ as follows:

$$
\widetilde{\delta} \tilde{\eta}=\frac{a}{b}\left(\delta \eta-\frac{n}{a^{2}} \xi[b]\right)=-\frac{n}{a b} \xi[b]
$$

and

$$
\begin{aligned}
\widetilde{\delta} \tilde{\Phi}= & \delta \Phi(X)+\frac{1-n}{b} g(\operatorname{grad} b, \varphi(X)) \\
& +\frac{a^{2}-b}{b} \eta(X) \delta \Phi(\xi)-\frac{1}{a} g(\operatorname{grad} a, \varphi(X)) .
\end{aligned}
$$

Let $(M, \varphi, \xi, \eta, g)$ be a quasi-Sasakian manifold, $b$ be a positive constant and $a$ be a function such that grada $=\xi[a] \xi$. After a generalized D-conformal deformation, the new covariant derivative is

$$
\tilde{\nabla}_{X} Y=\nabla_{X} Y+\frac{1}{a} \xi[a] \eta(X) \eta(Y) \xi+\frac{a^{2}-b}{b}\left(\eta(Y) \nabla_{X} \xi+\eta(X) \nabla_{Y} \xi\right)
$$

Moreover, by direct calculation, one can get

$$
\tilde{R}(X, \tilde{\xi}) \tilde{\xi}=\frac{1}{b} R(X, \xi) \xi-\frac{1}{a b} \xi[a] \nabla_{X} \xi-\frac{a^{2}-b}{b^{2}} \nabla_{X} \xi \xi
$$

and

$$
\begin{aligned}
\tilde{R}\left(X, \tilde{e}_{i}\right) \tilde{e}_{i}= & \frac{1}{b} R\left(X, e_{i}\right) e_{i}+\frac{a^{2}-b}{b^{2}} \eta(X)\left(\nabla_{\nabla_{e_{i}} e_{i}} \xi-\nabla_{e_{i}} \nabla_{e_{i}} \xi\right) \\
& +3 \frac{a^{2}-b}{b^{2}} g\left(e_{i}, \nabla_{X} \xi\right) \nabla_{e_{i}} \xi .
\end{aligned}
$$


Hence, we obtain new Ricci operator $\tilde{Q}$ and scalar curvature $\tilde{S}$ as

$$
\begin{gathered}
\tilde{Q}(X)=\frac{1}{b} Q(X)-\frac{1}{a b} \xi[a] \nabla_{X} \xi+\frac{a^{2}-b}{b^{2}} \eta(X) \sum_{i=1}^{2 n}\left(\nabla_{\nabla_{e_{i}} e_{i}} \xi-\nabla_{e_{i}} \nabla_{e_{i}} \xi\right) \\
+2 \frac{a^{2}-b}{b^{2}} \nabla_{\nabla_{X} \xi} \xi, \\
\tilde{S}=\frac{1}{b} S-\frac{a^{2}-b}{b^{2}} \sum_{i=1}^{2 n} g\left(\nabla_{e_{i}} \xi, \nabla_{e_{i}} \xi\right) .
\end{gathered}
$$

Example 2. Let $M$ be a seven dimensional 3-Sasakian manifold. Since this manifold is Sasakian $\left(\mathcal{C}_{6}\right)$, it is in particular quasi-Sasakian $\left(\mathcal{C}_{6} \oplus \mathcal{C}_{7}\right)$. It is known that its scalar curvature is 42 and also $\nabla_{X} \xi=-\varphi(X)$ for all vector fields $X$. For definition and properties of 3-Sasakian manifolds, see [8].

Let $(M, \varphi, \xi, \eta, g)$ be one of the three Sasakian structures on a seven dimensional 3-Sasakian manifold and assume that a generalized D-conformal deformation is applied to this structure. Note that by Theorem [3], the deformed structure is also quasi-Sasakian. Now we calculate the new scalar curvature $\tilde{S}$ of the deformed manifold. Since

$$
g\left(\nabla_{e_{i}} \xi, \nabla_{e_{i}} \xi\right)=g\left(\varphi\left(e_{i}\right), \varphi\left(e_{i}\right)\right)=g\left(e_{i}, e_{i}\right)-\eta\left(e_{i}\right) \eta\left(e_{i}\right),
$$

from the Equation (18),

$$
\tilde{S}=\frac{6}{b}\left(8-\frac{a^{2}}{b}\right) .
$$

Let $a$ and $b$ be positive constants satisfying $\frac{1}{b}\left(8-\frac{a^{2}}{b}\right)=\frac{1}{6}$ or $\frac{1}{b}\left(8-\frac{a^{2}}{b}\right)=-\frac{1}{6}$. Then, the Equation (19) implies $\tilde{S}=1$ or $\tilde{S}=-1$. Thus the new quasi-Sasakian manifold is locally isometric to the sphere or the hyperbolic space, respectively.

On the other hand, if positive constants $a$ and $b$ are chosen $a s a^{2}=8 b$, then $\tilde{S}=0$ and the new quasi-Sasakian manifold has zero scalar curvature.

\section{Generalized D-Conformal Deformations of $\beta$-Kenmotsu Manifolds}

An almost contact metric manifold $(M, \varphi, \xi, \eta, g)$ is called $\beta$-Kenmotsu manifold, if the relation

$$
\left(\nabla_{X} \varphi\right) Y=\beta(g(\varphi(X), Y) \xi-\eta(Y) \varphi(X)),
$$

is satisfied, where $\beta$ is a smooth function on $M$. It is known that if $(M, \varphi, \xi, \eta, g)$ is a $\beta$-Kenmotsu manifold, then the equation

$$
g\left(\nabla_{X} \xi, Y\right)=g\left(\nabla_{Y} \xi, X\right)
$$

is satisfied.

Lemma 4. Consider a generalized D-conformal deformation of an almost contact metric structure such that $g\left(\nabla_{X} \xi, Y\right)=g\left(\nabla_{Y} \xi, X\right)$, where $a$ and $b$ positive functions. After a generalized D-conformal deformation, the new Levi-Civita covariant derivative is

$$
\begin{aligned}
\widetilde{\nabla}_{X} Y= & \nabla_{X} Y+\frac{\left(a^{2}-b\right)}{b} g\left(\nabla_{X} \xi, Y\right) \xi+\frac{1}{2 b}(X[b] Y+Y[b] X) \\
& +\frac{1}{a}(X[a] \eta(Y)+Y[a] \eta(X)) \xi-\frac{1}{2 b}(X[b] \eta(Y)+Y[b] \eta(X)) \xi \\
& +\frac{a}{b} \eta(X) \eta(Y) \varphi^{2}(\operatorname{grad} a)-\frac{1}{a} \xi[a] \eta(X) \eta(Y) \xi \\
& -\frac{1}{2 a^{2}} \xi[b] g(\varphi(X), \varphi(Y)) \xi+\frac{1}{2 b} g(\varphi(X), \varphi(Y)) \varphi^{2}(\operatorname{grad} b) .
\end{aligned}
$$

Then, we obtain the following lemma: 
Lemma 5. Let $(M, \varphi, \xi, \eta, g)$ be a $\beta$-Kenmotsu manifold. Consider a generalized D-conformal deformation on $M$ where $a$ and $b$ are positive functions. Then

$$
\tilde{g}\left(\widetilde{\nabla}_{X} \tilde{\xi}, Y\right)=\tilde{g}\left(\widetilde{\nabla}_{Y} \tilde{\xi}, X\right)
$$

if and only if the function a depends only on the direction of $\xi$, that is grada $=\xi[a] \xi$.

Proof. First by taking $Y=\xi$ in (21), we get

$$
\begin{aligned}
g\left(\tilde{\nabla}_{X} \xi, Y\right)= & g\left(\nabla_{X} \xi, Y\right)+\frac{1}{2 b} \xi[b] g(\varphi(X), \varphi(Y))+\frac{1}{a} X[a] \eta(Y) \\
& -\frac{a}{b} \eta(X) Y[a]+\frac{a}{b} \xi[a] \eta(X) \eta(Y)
\end{aligned}
$$

and

$$
\eta\left(\tilde{\nabla}_{X} \tilde{\xi}\right)=\frac{1}{a} X[a] .
$$

Then, by the definition of the generalized D-conformal deformation and the Equations (22) and (23), we have

$$
\begin{aligned}
\tilde{g}\left(\widetilde{\nabla}_{X} \tilde{\xi}, Y\right) & =b g\left(\tilde{\nabla}_{X} \tilde{\xi}, Y\right)+\left(a^{2}-b\right) \eta\left(\tilde{\nabla}_{X} \tilde{\xi}\right) \eta(Y) \\
& =-\frac{b}{a^{2}} X[a] \eta(Y)+\frac{b}{a} g\left(\tilde{\nabla}_{X} \xi, Y\right)-\frac{\left(a^{2}-b\right)}{a^{2}} X[a] \eta(Y)+\frac{\left(a^{2}-b\right)}{a} \eta\left(\tilde{\nabla}_{X} \tilde{\xi}\right) \eta(Y) \\
& =\frac{b}{a} g\left(\nabla_{X} \xi, Y\right)+\frac{1}{2 a} \xi[b] g(\varphi(X), \varphi(Y))-Y[a] \eta(X)+\xi[a] \eta(X) \eta(Y) .
\end{aligned}
$$

Let

$$
\tilde{g}\left(\widetilde{\nabla}_{X} \tilde{\xi}, Y\right)=\tilde{g}\left(\widetilde{\nabla}_{Y} \tilde{\xi}, X\right)
$$

The Equation (24) implies

$$
\eta(Y) X[a]=\eta(X) Y[a]
$$

Take $Y=\xi$ in (25) to obtain

$$
X[a]=\eta(X) \xi[a],
$$

that is

$$
\operatorname{grad} a=g(\operatorname{grad} a, \xi) \xi .
$$

Conversely, let grad $a=g(\operatorname{grad} a, \xi) \xi$. Then, the term $-Y[a] \eta(X)+\xi[a] \eta(X) \eta(Y)$ of the Equation (24) vanishes and thus $\tilde{g}\left(\widetilde{\nabla}_{X} \tilde{\xi}, Y\right)=\tilde{g}\left(\widetilde{\nabla}_{Y} \tilde{\xi}, X\right)$ is obtained.

For any $\beta$-Kenmotsu manifold, we know that $d \eta=0$. After deformation, derivation of $\widetilde{\eta}$ is obtained as:

$$
d \widetilde{\eta}(X, Y)=a d \eta(X, Y)+X[a] \eta(Y)-Y[a] \eta(X) .
$$

The new Levi-Civita covariant derivative of $\tilde{\Phi}$ is

$$
\begin{aligned}
\left(\widetilde{\nabla}_{X} \widetilde{\Phi}\right)(Y, Z)= & b\left(\nabla_{X} \Phi\right)(Y, Z)-\frac{1}{2} Y[b] \Phi(X, Z)+\frac{1}{2} Z[b] \Phi(X, Y) \\
& a \eta(X)(\eta(Y) \varphi(Z)[a]-\eta(Z) \varphi(Y)[a]) \\
& +\frac{1}{2} g(\varphi(X), \varphi(Y)) \varphi(Z)[b]-\frac{1}{2} g(\varphi(X), \varphi(Z)) \varphi(Y)[b] .
\end{aligned}
$$

Note that the following theorem can be deduced from Lemma 4.1 of [4]. In [4], first the new Levi-Civita covariant derivative is calculated under the restriction that $a$ and $b$ are positive functions depending on the direction of $\xi$ and then Lemma 4.1 in [4] is stated for trans-Sasakian manifolds 
by using this covariant derivative. In our study, however, we obtain the new Levi-Civita covariant derivative only by assuming that $d \eta=0$ (equivalently $g\left(\nabla_{X} \xi, Y\right)=g\left(\nabla_{Y} \xi, X\right)$ ) in Lemma 5 and then we state the following theorem.

Theorem 4. Let $(M, \varphi, \xi, \eta, g)$ be a $\beta$-Kenmotsu manifold, and consider a generalized D-conformal deformation with $a$ and $b$ positive functions. If grad $a=g(\operatorname{grad} a, \xi) \xi$ and $\operatorname{grad} b=\operatorname{g}(\operatorname{grad} b, \tilde{\xi}) \xi$, then $(M, \tilde{\varphi}, \tilde{\xi}, \tilde{\eta}, \tilde{g})$ is a $\tilde{\beta}$-Kenmotsu manifold, where

$$
\tilde{\beta}=\frac{1}{a} \beta+\frac{1}{2 a b} \xi[b] .
$$

Proof. One can easily calculate

$$
\begin{aligned}
\left(\widetilde{\nabla}_{X} \tilde{\varphi}\right)(Y)= & \widetilde{\nabla}_{X} \tilde{\varphi}(Y)-\tilde{\varphi}\left(\widetilde{\nabla}_{X} Y\right) \\
= & \left(\nabla_{X} \varphi\right)(Y)+\left(\frac{a^{2}-b}{a^{2}}\right) g\left(\nabla_{X} \xi, \varphi(Y)\right) \xi+\frac{1}{a} \varphi(Y)[a] \eta(X) \xi \\
& -\frac{1}{2 b} \varphi(Y)[b] \eta(X) \xi+\frac{1}{2 a^{2}} \xi[b] g(\varphi(X), Y) \xi-\frac{1}{2 b} g(\varphi(X), Y) \varphi^{2}(\operatorname{grad} b) \\
& +\frac{1}{2 b}(\varphi(Y)[b] X-Y[b] \varphi(X)) \\
& +\frac{a}{b} \eta(X) \eta(Y) \varphi(\operatorname{grad} a)+\frac{1}{2 b} g(\varphi(X), \varphi(Y)) \varphi(\operatorname{grad} b)
\end{aligned}
$$

Since $X[a] \eta(Y)=Y[a] \eta(X)$ for all vector fields $X$ and $Y$, taking grad $b=\xi[b] \xi$, then

$$
\begin{aligned}
\left(\widetilde{\nabla}_{X} \tilde{\varphi}\right)(Y)= & \frac{b}{a^{2}} \beta g(\varphi(X), Y) \xi+\frac{1}{2 a^{2}} \xi[b] g(\varphi(X), Y) \xi \\
& -\frac{1}{2 b} \xi[b] \eta(Y) \varphi(X)-\beta \eta(Y) \varphi(X) \\
= & \left(\frac{\beta}{a}+\frac{1}{2 a b} \xi[b]\right)\left(\frac{b}{a} g(\varphi(X), Y) \xi-a \eta(Y) \varphi(X)\right) \\
= & \left(\frac{\beta}{a}+\frac{1}{2 a b} \xi[b]\right)(\tilde{g}(\varphi(X), Y) \tilde{\xi}-\tilde{\eta}(Y) \varphi(X)) .
\end{aligned}
$$

Let $(M, \varphi, \tilde{\xi}, \eta, g)$ be a $\beta$-Kenmotsu manifold. Then, if $(M, \tilde{\varphi}, \tilde{\xi}, \tilde{\eta}, \tilde{g})$ is $\tilde{\beta}$-Kenmotsu, then from Lemma 5 , we obtain grad $a=\xi[a] \xi$. In addition, since $M$ is $\tilde{\beta}$-Kenmotsu, we have

$$
\left(\tilde{\nabla}_{X} \tilde{\varphi}\right)(Y)=\tilde{\beta}(\tilde{g}(\varphi(X), Y) \tilde{\zeta}-\tilde{\eta}(Y) \tilde{\varphi}(X)) .
$$

Take $Y=\xi$ in (27), then we obtain $\tilde{\beta}=\frac{1}{a} \beta+\frac{1}{2 a b} \xi[b]$. We have been unable to find any restriction on the function $b$.

If $(M, \varphi, \xi, \eta, g)$ is a $2 n+1$ dimensional $\beta$-Kenmotsu manifold, $a$ and $b$ positive functions such that grad $a=\xi[a] \xi$, grad $b=\xi[b] \xi$, after a generalized D-conformal deformation, we have

$$
\widetilde{\nabla}_{\tilde{\xi}} \tilde{\zeta}=\frac{1}{a b} \varphi^{2}(\operatorname{grad} a), \quad\left(\widetilde{\nabla}_{\tilde{e}_{i}} \tilde{\eta}\right)\left(\tilde{e}_{i}\right)=-\frac{1}{a} \eta\left(\nabla_{e_{i}} e_{i}\right)+\frac{1}{2 a b} \tilde{\xi}[a],
$$

and coderivations of $\eta$ and $\Phi$ are calculated as

$$
\widetilde{\delta} \tilde{\eta}=-\sum_{i=1}^{2 n}\left(\widetilde{\nabla}_{\tilde{e}_{i}} \tilde{\eta}\right)\left(\tilde{e}_{i}\right)=\frac{1}{a}\left(\delta \eta-\frac{n}{b} \xi[b]\right)
$$




$$
(\widetilde{\delta} \tilde{\Phi})(X)=\delta \Phi(X)+g\left(\operatorname{grad}\left(\ln \left(b^{n-1} a\right)\right), \varphi(X)\right) .
$$

In addition, by long direct calculation, the new scalar curvature $\tilde{S}$ is

$$
\begin{aligned}
\tilde{S}= & \frac{1}{b} S-2 \frac{a^{2}-b}{a^{2} b} g(Q(\xi), \xi)-\frac{n(2 n-3)}{2 a^{2} b^{2}} \xi[b]^{2}+\frac{4 n}{a^{3}} \xi[a] \beta \\
& -\frac{2 n}{a^{2} b} \xi[\xi[b]]+\frac{2 n}{a^{3} b} \xi[a] \xi[b]-\frac{2 n(2 n+1)}{a^{2} b} \xi[b] \beta+2 n(2 n-1) \frac{a^{2}-b}{a^{2} b} \beta^{2} .
\end{aligned}
$$

Author Contributions: All authors contributed equally.

Funding: No specific funding was received for this project.

Conflicts of Interest: The authors declare no conflict of interest.

\section{References}

1. Vaisman, I. Conformal changes of almost contact metric structures. In Geometry and Differential Geometry, Lecture Notes in Mathematics 792; Artzy, R., Vaisman, I., Eds.; Springer: Berlin, Germany, 1980; pp. 435-443.

2. Blair, D.E.; Oubina, J. A. Conformal and Related Changes of Metric on the product of Two Almost Contact Metric Manifolds. Publ. Mat. 1990, 34, 199-207. [CrossRef]

3. De, U.C.; Ghosh, S. D-Homothetic Deformation of Normal Almost Contact Metric Manifolds. Ukr. Math. J. 2013, 64, 1514-1530. [CrossRef]

4. Alegre, P.; Carriazo, A. Generalized Sasakian Space Forms and Conformal Changes of the Metric. Results Math. 2011, 59, 485-493. [CrossRef]

5. Chinea, D.; Gonzales, C. A Classification of Almost Contact Metric Manifolds. Ann. Mat. Pura Appl. 1990, 156, 15-36. [CrossRef]

6. Alexiev, V.; Ganchev, G. On the Classification of Almost Contact Metric Manifolds. In Mathematics and Education in Mathematics, Proceedings of 15th Spring Conference of UBM, Sunny Beach, Bulgaria, 6-9 April 1986; Professor Marin Drinov Academic Publishing House: Sofia, Bulgaria, 1986; pp. 155-161.

7. Blair, D.E. The Theory of Quasi-Sasakian Structures. J. Differ. Geom. 1967, 1, 331-345. [CrossRef]

8. Agricola, I.; Friedrich, T. 3-Saskian Manifolds in Dimension Seven, Their Spinors and $G_{2}$-Structures. J. Geom. Phys. 2010, 60, 326-332. [CrossRef]

(C) 2019 by the authors. Licensee MDPI, Basel, Switzerland. This article is an open access article distributed under the terms and conditions of the Creative Commons Attribution (CC BY) license (http://creativecommons.org/licenses/by/4.0/). 